\title{
Variability of GPS Units for Measuring Distance in Team Sport Movements
}

\author{
Denise Jennings, Stuart Cormack, Aaron J. Coutts, \\ Luke J. Boyd, and Robert J. Aughey
}

Purpose: To examine the difference in distance measured by two global positioning system (GPS) units of the same model worn by the same player while performing movements common to team sports. Methods: Twenty elite Australian football players completed two trials of the straight line movement (10, 20, 40 m) at four speeds (walk, jog, stride, sprint), two trials of the changes of direction (COD) courses of two different frequencies (gradual and tight), and five trials of a team sport running simulation circuit. To assess inter-unit variability for total and high intensity running (HIR) distance measured in matches, data from eight field players were collected in three Australian Hockey League (AHL) matches during the 2009 season. Each subject wore two GPS devices (MinimaxX v2.5, Catapult, Australia) that collected position data at $5 \mathrm{~Hz}$ for each movement and match trial. The percentage difference $\pm 90 \%$ confidence interval $(\mathrm{CI})$ was used to determine differences between units. Results: Differences $( \pm 90 \% \mathrm{CI})$ between the units ranged from $9.9 \pm 4.7 \%$ to $11.9 \pm 19.5 \%$ for straight line running movements and from $9.5 \pm 7.2 \%$ to $10.7 \pm 7.9 \%$ in the COD courses. Similar results were exhibited in the team sport circuit $(11.1 \pm 4.2 \%)$. Total distance $(10.3 \pm 6.2 \%)$ and HIR distance $(10.3 \pm 15.6)$ measured during the match play displayed similar variability. Conclusion: It is recommended that players wear the same GPS unit for each exercise session to reduce measurement error. The level of between-unit measurement error should be considered when comparing results from players wearing different GPS units.

Keywords: GPS, variability, precision

Global positioning system (GPS) technology is commonly used for the quantification of movement in team sports. ${ }^{1,2}$ Several studies have investigated the reliability

\footnotetext{
Denise Jennings is with the School of Sport and Exercise Science, Institute of Sport, Exercise and Active Learning (ISEAL), Victoria University, Melbourne, Australia, and the High Performance Unit, Essendon Football Club, Melbourne, Australia. Stuart Cormack is with the High Performance Unit, Essendon Football Club, Melbourne, Australia. Aaron J.Coutts is with the High Performance Unit, Essendon Football Club, Melbourne, Australia, and the School of Leisure, Sport \& Tourism, University of Technology, Sydney, Australia. Luke J. Boyd is with the School of Sport and Exercise Science, Institute of Sport, Exercise and Active Learning (ISEAL), Victoria University, Melbourne, Australia, and the Western Bulldogs Football Club, Melbourne, Australia. Robert J. Aughey is with the School of Sport and Exercise Science, Institute of Sport, Exercise and Active Learning (ISEAL), Victoria University, Melbourne, Australia, and the Western Bulldogs Football Club, Melbourne, Australia.
} 
and validity of GPS devices for measuring movements and velocities in different sporting activities. ${ }^{3-6}$ However, few have examined the between-unit variation with $5 \mathrm{~Hz}$ GPS. Quantification of between-unit measurement variation allows more accurate comparisons between players or bouts of activity. The aim of this study was to examine the differences between distance measures of two GPS units of the same model worn by the same player while performing team sport movements.

\section{Methods}

Twenty Australian football (AF) players (mean $[ \pm \mathrm{SD}]$ age, stature and body mass: $24 \pm 4 \mathrm{y}, 188 \pm 7 \mathrm{~cm}, 87 \pm 9 \mathrm{~kg}$, respectively) completed the trials. Eight male hockey players (mean $[ \pm$ SD] age, stature and body mass: $22 \pm 4 \mathrm{y}, 178 \pm 8 \mathrm{~cm}$ and $78 \pm 9 \mathrm{~kg}$, respectively) participated in the match play. All athletes gave written informed consent.

\section{Experimental Design}

The differences in distance measures obtained from two GPS units worn by the same subject were assessed during two trials each of straight line locomotion at four speeds and two change of direction (COD) courses $(40 \mathrm{~m})$. Between unit variability for high intensity running distance (HIR; running speed $>4.17 \mathrm{~m} \cdot \mathrm{s}^{-1}$ ) and total distance were made using a team sport running circuit $(140 \mathrm{~m})$ and during match play. A detailed explanation of procedures, satellite number and horizontal dilution of precision (HDOP) during data collection for trials can be found elsewhere. ${ }^{3}$ Data from eight field players was collected during three Australian Hockey League (AHL) matches during the 2009 season $(n=24)$. Each game was played in the afternoon at the same venue. The number of satellites and HDOP (mean \pm SD) during match play data collection for the match play were $8.5 \pm 0.5$ and $1.2 \pm$ $0.3 ; 7.8 \pm 0.4$ and $1.1 \pm 0.1$; and $8.4 \pm 0.6$ and $1.1 \pm 0.1$ respectively. In each trial, subjects wore two 5-Hz GPS units (MinimaxX, Team 2.5, Catapult Innovations, Scoresby, Australia) in a custom-made harness with units located on each scapula, approximately $25 \mathrm{~cm}$ apart.

\section{Statistical Analyses}

The percentage difference $\pm 90 \%$ CI was used to determine differences between units, using the formula (Unit B/Unit A) $\times 100 / 1$. Total distance and HIR were chosen as variables during the team sport circuit and match play as a result of previous work. ${ }^{3}$ Smallest worthwhile change (SWC) was calculated as $0.2 \times$ between-subject standard deviation.

\section{Results}

Between-unit variability $( \pm 90 \% \mathrm{CI}$ ) ranged from $9.9 \pm 4.7 \%$ to $11.9 \pm 19.5 \%$ for straight line running and $9.5 \pm 7.2 \%$ to $10.7 \pm 7.9 \%$ for COD courses (Table 1 ). Similar results were exhibited for total and HIR distance in the simulated team sport circuit and match play (Table 2). 


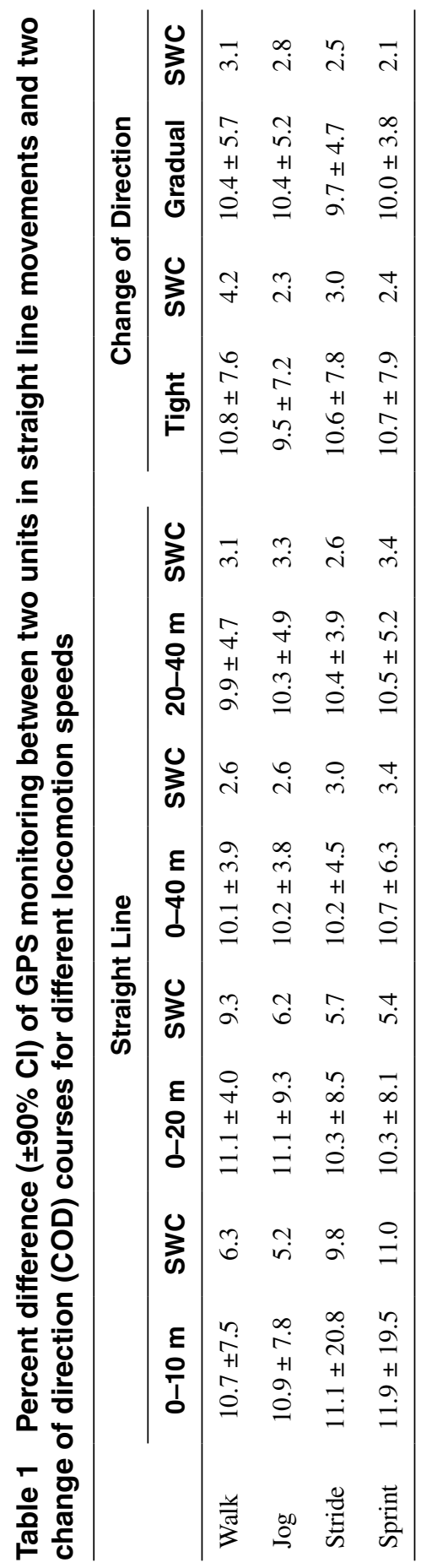


Table 2 Percent difference $( \pm 90 \% \mathrm{Cl})$ of GPS monitoring between two units during a team sport circuit and match play. Total distance and high intensity running distance (HIR; running speed $>4.17 \mathrm{~m} / \mathrm{s}$ ) are represented.

\begin{tabular}{lcccc}
\hline & Circuit & SWC $(\mathbf{m})$ & Match Play & SWC $(\mathbf{m})$ \\
\hline Total & $11.1 \pm 4.2$ & 1.5 & $10.3 \pm 6.2$ & 1.9 \\
HIR & $11.6 \pm 9.3$ & 3.2 & $10.3 \pm 15.6$ & 3.1 \\
\hline
\end{tabular}

\section{Discussion}

There was considerable variation in distance measured from two GPS units worn by the same player completing the same movement task. In addition, variation between the two GPS units was similar regardless of the movement pattern.

Only one study has reported between unit variability during field based team sport specific movements. ${ }^{5}$ In that study, $1-\mathrm{Hz}$ GPS units were used rather than the $5-\mathrm{Hz}$ units used here. In the present study, the magnitude of between-unit difference for the team sport circuit was $11.1 \pm 4.2 \%$, which is surprisingly greater than previously reported (coefficient of variation: $3.5-6.6 \%$ ). ${ }^{5}$ The differences in between unit variability reported in these studies may be due to the different software and hardware used to collect and treat data.

The level of between-unit variation was consistent (approx. 10\%) regardless of distance (total and HIR distance), movement speed and change of direction. However, there was less certainty in the measure at higher velocities that included a standing start, in line with our previous findings. ${ }^{3}$ The large CIs for $0-10 \mathrm{~m}$ stride and sprint demonstrate the uncertainty in GPS measurements over short distances. It is likely that this uncertainty is due to the rapid changes in velocity evident in movements when performed at higher speeds. This has important practical implications as when this between-unit difference is coupled with testretest error of GPS, ${ }^{3-6}$ the ability to detect small differences between players may be limited. ${ }^{3}$ Indeed, in this study, measurement error was often at least double the smallest worthwhile change in performance. To minimize this variability, it is recommended that the same unit be used for individual players during all exercise sessions, with consideration of the between-unit measurement error for comparisons between players.

Analysis of external loads (ie, total and HIR distance) provides valuable information regarding the physiological and performance requirements of team sport athletes. Sports scientists and coaches use distance measures from matches or training drills to assess work demands and compare performance of team sport athletes. These findings show that careful consideration should be given to the between-unit variability when interpreting results, particularly when comparing measures from players using different GPS units. Further work is required to reduce the between-unit variability before comparisons among players can be reliably performed. 


\section{References}

1. Roberts S, Trewartha G, Stokes K. A comparison of time-motion analysis methods for field-based sports. Int J Sports Physiol Perform. 2006;1:388-399.

2. Dobson B, Keogh JWL. Methodological issues for the application of time-motion analysis research. Strength Condit J. 2007;29:48-55.

3. Jennings D, Cormack S, Coutts AJ, Boyd L, Aughey RJ. The validity and reliability of GPS units for measuring distance in team sport specific running patterns. Int J Sports Physiol Perform. 2010;5:328-341.

4. Petersen C, Pyne D, Portus M, Dawson B. Validity and reliability of GPS units to monitor cricket-specific movement patterns. Int J Sports Physiol Perform. 2009;4:381-393.

5. Coutts AJ, Duffield R. Validity and reliability of GPS units for measuring movement demands of team sports. J Sci Med Sport. 2010;13:133-135.

6. Duffield R, Reid M, Baker J, Spratford W. Accuracy and reliability of GPS devices for measurement of movement patterns in confined spaces for court-based sports. $J$ Sci Med Sport. 2010;13:523-525. 